\title{
Comparison of a magnetic retrieval device vs. flexible cystoscopy for removal of ureteral stents in renal transplant patients: A randomized controlled trial
}

Anil Kapoor, MD ${ }^{1,2,3}$; Jason Akerman, MD ${ }^{1,4}$; Emily C.L.Wong, BHSc ${ }^{1}$; Gaurav Vasisth, MD ${ }^{1}$; Fadil Hassan, MD ${ }^{1}$; Camilla Tajzler, BA ${ }^{1}$; Kevin Piercey, MD ${ }^{1,2,3}$; Jen Hoogenes, MS, MSc, $\mathrm{PhD}^{1,2}$; Shahid Lambe, $\mathrm{MD}^{1,2,3}$

${ }^{1}$ Department of Surgery, Division of Urology, McMaster University, Hamilton, ON, Canada; ${ }^{2}$ McMaster Institute of Urology, St. Joseph's Healthcare Hamilton, Hamilton, ON, Canada; ${ }_{3}^{3}$ uravinski Cancer Centre, Juravinski Hospital, Hamilton, ON, Canada; ${ }^{4}$ Department of Urology, University of North Carolina, Chapel Hill, NC, United States

Cite as: Can Urol Assoc J 2020 July 27; Epub ahead of print. http://dx.doi.org/10.5489/cuaj.6684

Published online July 27, 2020

$* * *$

\section{Abstract}

Introduction: Placement of a ureteral stent at the time of renal transplantation can reduce complications when compared to non-stented anastomoses. Removal by flexible cystoscopy can be associated with discomfort, risk for infection, and high costs. New magnetic stents offer a means of bypassing cystoscopy by use of a magnetic retrieval device. Our objective was to compare clinical and cost-related outcomes of conventional and magnetic stents in patients undergoing deceased donor renal transplantation.

Methods: Patients were randomized to receive either a conventional or a Black-Star ${ }^{\circledR}$ magnetic stent. Clinical, procedural, and cost outcomes were assessed, and the Ureteral Stent Symptom Questionnaire (USSQ) was administered with the stent in situ and after stent removal. All variables were compared between groups.

Results: Forty-one patients were randomized to conventional $(n=19)$ or Black-Star $(n=22)$ stent. The total time for stent removal under cystoscopy was significantly longer compared to Black-Star removal $(6.67 \pm 2.47$ and $4.80 \pm 2.21$ minutes, respectively; $\mathrm{p}=0.019)$. No differences were found in the USSQ domains between groups. Rates of urinary tract infections and surgical complications between groups were similar. Stent removal was well-tolerated in both groups. Black-Star stent use resulted in a cost savings of \$304.02 Canadian dollars (CAD) per case.

Conclusions: USSQ scores suggest that stent removal with the Black-Star magnetic stent is as equally well-tolerated as flexible cystoscopy by renal transplant patients. Black-Star stent removal was significantly faster than conventional stents. No differences in discomfort, infection rate, or complication rate were found. Use of the Black-Star stent resulted in an estimated annual savings of $\$ 27360 \mathrm{CAD}$ at our centre. 


\section{Introduction}

Renal transplantation is the treatment of choice for end-stage renal disease. Intraoperatively, a stent is placed in the transplant ureter between the renal pelvis and the bladder to reduce complications. The advantages of a stented anastomosis include continuous decompression of the ureter, reduced anastomotic tension, less kinking of the ureter, and maintaining a patent lumen against edema or external compression. ${ }^{1,2}$ Post-operatively, the use of a ureteral stent has been shown to reduce the incidence of urinary leaks and ureteral stenosis. These complications can contribute to increased patient morbidity, graft loss, and prolonged hospital stay in up to 3-9\% of renal transplant cases. ${ }^{1-4}$

The placement of a ureteral stent is not always without side effects. Stents have been associated with an increase in the number and severity of urinary tract infections (UTIs), intermittent hematuria, stent migration, and encrustation of the outer surface of the stent. ${ }^{5}$ Removal of a ureteral stent, typically done via flexible cystoscopy, can cause pain and distress for patients. Cystoscopy itself is often associated with discomfort or pain to the patient (38\%), transient urinary frequency $(15 \%)$, urgency $(14 \%)$, and/or urinary tract infection $(8 \%){ }^{6}$ The Black-Star ${ }^{\circledR}$ double-J ureteral stent with magnetic retrieval device (Urotech, Achenmühle, Germany) was approved by Health Canada in 2017 and offers a means of eliminating the need for flexible cystoscopy for stent removal, which can be done outside of the cystoscopy suite. Magnetic ureteral stents were first proposed nearly thirty years ago; ${ }^{7}$ however, patient discomfort and poor magnetization limited their uptake early on. Recent advancements in materials and stent design have since addressed these concerns. ${ }^{8,9}$ Using a small biocompatible magnet attached to a polyurethane body (Figure 1), the Black-Star® stent has shown reproducible success in patients post-ureteroscopy without added patient discomfort while affirming that retrieval is simple procedure (Figure 2). ${ }^{10}$

The objectives of this study were to evaluate clinical, procedural, and cost-related outcomes relative to the use of the Black-Star ${ }^{\circledR}$ stent and retrieval device versus a traditional ureteral stent with cystoscopy removal in deceased-donor renal transplant patients at a single centre. An additional aim was to determine the feasibility of conducting a larger randomized controlled trial.

\section{Methods}

This was a prospective randomized controlled trial at a single transplant centre in Ontario, Canada. Research ethics board approval was obtained by our affiliated academic institution. All patients meeting inclusion criteria in our transplant centre were asked to participate in the study between May 2017 and April 2018. Following the informed consent procedure, patients were randomly allocated to one of two study arms: magnetic ureteral stent (Black-Star®) (intervention) or conventional ureteral stent (controls). Once informed consent was gained, randomization was conducted using a random number generator via Research Randomizer® (www.randomizer.org) and participants were assigned a unique study identification number. Neither patients nor surgeons were blinded to group allocation. All patient identifiers were 
removed for analysis and confidentiality was maintained.

\section{Study population}

Prior to gaining informed consent, patients were screened for inclusion and exclusion criteria. Inclusion criteria were comprised of 1) patients were at least 18 years of age at the time of recruitment and capable of giving informed consent, and 2) patients were scheduled for deceased-donor renal transplant surgery at our centre. Patients were excluded if they were undergoing living donor renal transplantation, as at our centre, live donor recipients receive stents based on surgeon discretion only.

\section{Outcome measures}

The primary outcome measure was patient comfort of the stent in situ and after removal using the validated Ureteral Stent Symptom Questionnaire (USSQ), which was developed by the Bristol Urological Institute (Southmead Hospital, Bristol, UK). ${ }^{11}$ Secondary outcome measures included 1) stent retrieval time in minutes; 2) infection rate (number of urine culture positive tests with stent in situ and after removal); 3) cost-effectiveness in Canadian dollars (CAD), including cost of disposables, sterilization of instruments, use of cystoscopy suite, and nursing and physician charges (mean cost per procedure); and 4) to determine the feasibility of conducting a larger randomized controlled trial.

\section{Black-Star $^{\circledR}$ stent}

The Black-Star® stent is designed to allow for easy removal using a magnet attached to the distal end of the stent (Figure 2). In this study, a 10cm 4.8Fr Black-Star ${ }^{\circledR}$ stent was used. Removal of the stent was conducted using a $9 \mathrm{Fr}, 40 \mathrm{~cm}$ retrieval device. The catheter-like retrieval device is composed of soft polyurethane that features a $30^{\circ}$ Tiemann tip which facilitates interaction between the magnetic components of the stent and retrieval device (Figure 1). This allows the stent to be removed per urethra without the need for cystoscopy. Black-Star® stents were provided for this study by Red Leaf Medical, Inc. (Mississauga, ON, Canada).

\section{Data collection and analysis}

Prior to surgery, a series of baseline characteristics including patient demographic information, clinical history, and medical comorbidities was collected. Intraoperatively, all major and minor complications and any anatomical variants (e.g., number of ureters) were noted. Total surgical time was recorded, as well as any difficulties with placement of the stent or additional operative time due to stent placement.

All participants were followed from the time of surgery to 1week post-stent removal. The USSQ was completed at 4 weeks post-renal transplantation ("stent in situ" questionnaire) and at 1 week following stent removal ("post-stent" questionnaire). ${ }^{11}$ Both questionnaires are comprised of domains for urinary issues, pain index, general health, work performance, and sexual matters. Each domain can be scored and evaluated separately from the overall USSQ 
score. The questionnaires also contain a global quality of life score and an option for patients to report any additional problems related to the stent, as well as a space for free-text comments. Details of the validation and scoring of the USSQ are described in Joshi et al. (2003). ${ }^{11} \mathrm{~A}$ urinalysis and urine culture were collected 1-week post stent removal to assess the rate of UTIs in each group.

Data analysis was conducted using descriptive statistics, chi-squared tests, and independent samples $t$-tests for between groups analysis. Statistical significance was set at $\alpha=$ 0.05. All analyses were performed using SPSS version 26 (IBM SPSS Statistics, Armonk, New York, USA).

\section{Study procedures}

All cases were performed by one of three transplant surgeons at our centre, using the identical surgical approach. After back table preparation of the graft, extraperitoneal access was achieved using a modified Gibson's incision. Reconstruction of the urinary tract was done via urethrovesical anastomosis (Lich-Gregoir technique) using either the Black-Star ${ }^{\circledR}$ double J stent or a conventional $12 \mathrm{~cm} 4.8 \mathrm{Fr}$ double J stent. The placement of each stent was performed over a guidewire.

Patients randomized to the conventional stent group were seen in follow-up for stent removal via flexible cystoscopy at 6 weeks post-transplant. Removal was conducted by one of two renal transplant fellows at our centre. Patients were brought to the cystoscopy suite where the stents were removed under sterile technique. All patients received local anesthetic in the form of 11cc Instillagel@ (Farco-Pharma GmbH, Cologne, Germany), a lidocaine and chlorhexidine gel administered per urethra prior to insertion of the flexible cystoscope. A flexible stent grasper was inserted through the working port of the cystoscope, allowing the stent to be removed. All cystoscopes were $16.2 \mathrm{Fr}$ size. Time was recorded from when the patient entered the cystoscopy suite until the stent was removed. Patients were asked to state whether the removal was comfortable (yes/no).

Patients randomized to the Black-Star® group underwent stent removal in our outpatient transplant clinic by one of two renal transplant fellows, also at 6 weeks posttransplant. Under sterile technique, patients were given 11cc Instillagel@ local anesthetic followed by insertion of the magnetic retrieval device. This is done blindly and requires contact between the retrieval device and magnetic portion of the stent. Time was recorded from when the patient entered the room until the stent was removed. If a first pass of the retrieval device failed to remove the stent, a second pass was performed. Patients were then asked to state whether the removal was comfortable (yes/no).

\section{Cost analysis}

A cost analysis was performed to evaluate the cost per case between the Black-Star® and conventional stent. Black-Star ${ }^{\circledR}$ costs included the price of the stent and retrieval device, as well as supplies used at the time of removal (Instillagel@). Conventional stent costs included the cost of the stent, supplies (drapes, local anesthetic, irrigation, and tubing), processing costs 
of the flexible cystoscope, surgeon and nursing fees, and facility fees at our cystoscopy centre.

\section{Results}

A total of 41 patients were recruited and randomized, with 22 patients in the Black-Star® group and 19 in the conventional stent group. In the Black-Star® group, one patient was admitted to the intensive care unit for an unrelated medical event prior to having their stent removed. This patient's stent was removed at the bedside in the intensive care unit. Additionally, one Black-Star ${ }^{\circledR}$ patient required stent removal via flexible cystoscopy after failure to remove the stent using the magnetic retrieval device. This patient had prolonged stent placement due to a post-operative anastomotic leak which resulted in significant encrustation of the stent. The stent was successfully removed via flexible cystoscopy and the patient's post-stent questionnaire data were not included in the analysis.

Demographic and baseline characteristics are reported in Table 1. There was no significant difference between groups in age, sex, medical comorbidities, or baseline lower urinary tract symptoms. Urinary Index scores on the USSQ with stent in situ were not significantly different between groups, with a mean score of 21.48/56 ( \pm 5.47$)$ in the BlackStar ${ }^{\circledR}$ group and $22.47 / 56( \pm 3.89, \mathrm{p}=0.515)$ in the conventional stent group (for the Urinary Index domain, a lower score indicates fewer symptoms) (Table 2). Analysis of the other domains of the USSQ also showed no significant difference between groups with the stent in situ (Table 2). Mean USSQ scores for the Urinary Index 1-week post-stent removal were $21.75 / 56( \pm 4.06)$ in the Black-Star® group and $20.64 / 56( \pm 4.06 ; \mathrm{p}=0.481)$. This was consistent for each domain in the post-stent USSQ (Table 2).

The rate of UTIs based on positive urine culture collected 1-week post-stent removal, were not significantly different between groups, with 2 positive cultures in the Black-Star® group and 4 in the conventional stent group $(p=0.374)$. Six patients in the conventional stent group reported discomfort at the time of stent removal compared to 2 patients in the BlackStar ${ }^{\circledR}$ group $(\mathrm{p}=0.213)$. The Black-Star ${ }^{\circledR}$ stents required significantly less time for removal compared to the conventional stents. Mean time for stent removal with the Black-Star ${ }^{\circledR}$ magnetic retrieval device was $4.80 \pm 2.21$ minutes compared to $6.67 \pm 2.47$ minutes for conventional stent removal with flexible cystoscopy $(\mathrm{p}=0.019)$.

Upfront costs for the Black-Star ${ }^{\circledR}$ stent were higher than the conventional ureteral stent (Table 3). The use of the Black-Star® stent resulted in a cost savings by avoiding the need for a post-operative flexible cystoscopy. The estimated cost of a single conventional stent removal with flexible cystoscopy at our centre was $\$ 429.28$ Canadian dollars (CAD). When this is accounted for, the Black-Star® stent resulted in cost savings of \$304.02 CAD per case.

\section{Discussion}

Flexible cystoscopy has been associated with patient discomfort, pain, and anxiety, particularly among younger men. ${ }^{12-14}$ Several studies have evaluated methods of reducing 
patient distress at the time of flexible cystoscopy. ${ }^{15,16}$ Modifications to ureteral stents have been developed with the aim of eliminating the need for cystoscopy altogether. Recent advancements include magnetic ureteral stents that do not require cystoscopy for their removal, and biodegradable stents that require no removal at all. ${ }^{17-19}$ Our study adds to the published data on the Black-Star ${ }^{\circledR}$ magnetic ureteral stent as a well-tolerated alternative to conventional ureteral stents; ${ }^{17-20}$ however, our study further contributes to the literature base as the use of these stents were within the deceased-donor renal transplantation setting.

Existing published data have focused primarily on the use of magnetic stents among pediatric and post-ureteroscopy patients. ${ }^{17,21,22}$ In our study, deceased-donor renal transplantation patients tolerated the Black-Star ${ }^{\circledR}$ stent without any major complications. Intraoperative reports indicated that the Black-Star® stents were easily placed with no increase in operative times. All deceased-donor renal transplantation patients at our centre receive a ureteral stent at the time of surgery and many of them undergo pre-operative evaluation of their lower urinary tract with cystoscopy. This allowed many patients to compare their experience with a magnetic stent retrieval device to their prior experience with flexible cystoscopy. The superior location of the stent within the bladder of transplant patients (compared to the usual location of a native ureteral orifice) did not cause any difficulty with removal. We were not able to demonstrate any significant differences in patient comfort with stent removal via a magnetic retrieval device compared to flexible cystoscopy. This is not consistent with previously studies which showed that the Black-Star® stent was preferred to flexible cystoscopy with respect to patient comfort during the removal. ${ }^{17,20}$ Rasseweiler et al., found that patients who had a Black-Star ${ }^{\circledR}$ stent placed following ureterorenoscopy reported significantly less pain at the time of removal compared to those with a conventional stent using a visual analog scale. ${ }^{17}$ Likewise, O'Connell et al. found that among patients who underwent a prior cystoscopy, $71 \%$ preferred the magnetic retrieval device of the Black-Star ${ }^{\circledR}$ stent. ${ }^{20}$ Our study's patients responded to the yes/no question "Was stent retrieval comfortable?" to assess discomfort at the time of stent removal. We feel that this binary question limited our ability to detect more subtle differences in patient discomfort and/or distress at the time of stent removal between groups. The use of a visual analog scale, as reported in other Black-Star® studies, may have yielded more useful and consistent results and will be considered in future research.

Although the Black-Star® stent was well-tolerated in our study, there are important considerations for patient selection. One patient in the Black-Star ${ }^{\circledR}$ group required removal of their stent via flexible cystoscopy, which was delayed well beyond the 6-week mark due to an anastomotic urine leak. As a result of stent encrustation, we were unable to remove the BlackStar® using its retrieval device. As this is a rare complication, we feel that the Black-Star® stent remains a viable option for deceased-donor renal transplantation patients, as the majority will have their stent removed before significant encrustation occurs. In addition to stent encrustation, the presence of a large median lobe has been reported as a barrier to removal using the magnetic retrieval device. ${ }^{17}$ This may be a pre-operative consideration for stent 
selection in patients with a documented occlusive median lobe.

By eliminating flexible cystoscopy, we have shown that in deceased-donor renal transplant patients, magnetic ureteral stents can be removed in less time with decreased costs when compared to conventional stent removal with flexible cystoscopy. In our study, time was recorded from when patients entered the room to the time of stent removal. The removal time for conventional stents included the turnover and instrument preparation factors unique to the cystoscopy suite, while the Black-Star ${ }^{\circledR}$ removal was conducted in an outpatient clinic room. However, in observing the significant time differences between the conventional and Black-Star ${ }^{\circledR}$ removal $(6.67 \pm 2.47$ and $4.80 \pm 2.21$ minutes, respectively; $\mathrm{p}=0.019)$, it is important to consider the overall time saved by bypassing the cystoscopy suite for magnetic stent removal.

The cost savings observed in our study are consistent with previous reports which had estimated savings of $€ 100-€ 810$ (approximately $\$ 155-\$ 1245$ CAD). ${ }^{17,20}$ Despite a higher upfront cost of the Black-Star® versus a conventional stent (\$119 vs. \$64.85 CAD, respectively), the use of the Black-Star® stent resulted in considerable cost savings by avoiding the physician, nursing, and processing fees of a flexible cystoscopy (Table 3). At our centre, the use of the Black-Star ${ }^{\circledR}$ stent in all deceased-donor renal transplantation alone would generate an annual cost savings of approximately \$27,360 CAD.

Our study is limited by its small sample size. However, this study confirms the feasibility of conducting a larger randomized controlled trial to analyze clinical and costrelated differences between the use of conventional and Black-Star® magnetic stents in deceased-donor renal transplantation patients. The addition of a visual analog scale or Likerttype pain scale at the time of stent removal may increase the ability to more accurately detect differences in pain and discomfort with flexible cystoscopy compared to the use of a magnetic retrieval device. Furthermore, UTIs were estimated based on culture results alone and were not confirmed by patient symptoms. This may have led to some asymptomatic bacteriuria being labeled as a UTI. Given the low overall rate of culture positive results, we feel this is unlikely to have changed our results.

\section{Conclusions}

The Black-Star® magnetic stent and retrieval device offers an alternative to conventional ureteral stents in deceased-donor renal transplantation patients. In our study, the Black-Star ${ }^{\circledR}$ stent was well-tolerated with no increase in major complications or patient discomfort when compared to the conventional stent. Despite its higher upfront costs, the use of the BlackStar® stent and retrieval device has the potential to offer considerable cost savings over conventional stents by eliminating the need for post-operative cystoscopy. Further study with larger, multicentre randomized controlled trials should be conducted to determine the clinical and monetary value of using magnetic stents, such as the Black-Star ${ }^{\circledR}$, versus conventional ureteral stents in renal transplant patients. 


\section{References}

1. Mangus RS, Haag BW. Stented versus non-stented extravesical ureteroneocystostomy in renal transplantation: a meta-analysis. Am J Transplant 2004; 4:1889-96.

2. Kumar A, Kumar R, Bhandari M. Significance of routine JJ stenting in living related renal transplantation: A prospective, randomized study. Transplant Proc 1998; 30:2995-7.

3. DuBay DA, Moyer AS, Englesbe MJ, et al. Predictive factors for ureteral complications after renal transplantation. Am J Transplant 2005; 5:201.

4. Englesbe MJ, Dubay DA, Gillespie BW, et al. Risk factors for urinary complications in renal transplantation. Am J Transplant 2007; 7:1536- 41.

5. Wilson $\mathrm{CH}$, Rix DA, and Manas DM. Routine intraoperative ureteric stenting for kidney transplant recipients. Cochrane Database Syst Rev 2013;6:CD004925.

6. Theckumparampil N, Elsamra SE, Carons A, et al. Symptoms after removal of ureteral stents. J Endourol 2015;29: 246-52.

7. Macaluso JN, Deutsch JS, Goodman JR, et al. The use of the Magnetic double-J ureteral stent in urological practice. J Urol 1989; 142:701-3.

8. Taylor WN, McDougall IT. Minimally invasive ureteral stent retrieval. J Urol 2002; 168:2020-23.

9. Chew BH, Lange D. Advances in ureteral stent development. Curr Opin Urol 2016; 26:277-82.

10. Wang J, Feng J, Hu W, Song Y, Xu X, Fan M. Preclinical evaluation of a newly designed ureteral stent and magnetic retrieval catheter for minimally invasive stent removal. Urology 2014; 84:960-6.

11. Joshi HB, Newns N, Stainthorpe A, et al. Ureteral stent symptom questionnaire: Development and validation of a multidimensional quality of life measure. J Urol 2003; 169:1060-4.

12. Taghizadeh AK, El Madani A, Gard PR, et al. When does it hurt? Pain during flexible cystoscopy in men. Uro Int 2006; 76:301-3.

13. Castelejin NF, Vriesema JL, Stomps SP, et al. The effect of office-based flexible and rigid cystoscopy on pain experience in female patients. Investig Clin Urol 2017; 58:48-53.

14. Burke DM, Shackley DC, O'Reilley PH. The community-based morbidity of flexible cystoscopy. BJU Int 2002; 89:347-49.

15. Zhang ZS, Wang XL, Zeng SX, et al. Pressure makes pleasure: A preliminary study of increasing irrigation pressure of flexible cystoscopy improves male patient comfort by an easy way. $J$ Endourol 2015; 29:1361-5.

16. Losco G, Antoniou S, Mark S. Male flexible cystoscopy: does waiting after insertion of topical anesthetic lubricant improve patient comfort. BJU Int 2011; 108:42-4.

17. Rassweiler MC, Michel MS, Ritter M, et al. Magnetic ureteral stent removal without cystoscopy: A randomized controlled trial. J Endoruol 2017; 31:762-6.

18. Barros AA, Oliveira C, Reis RL, et al. Ketoprofen-eluting biodegradable ureteral stents by CO2 impregnation: in vitro study. Int J Pharm 2015; 495:651-9.

19. Barros AA, Rita A, Duarte C, et al. Bioresorbable ureteral stents from natural origin 
polymers. J Biomed Mater Res B Appl Biomater 2015; 103:608-17.

20. O'Connell L, Broe MP, Rooney D, et al. Magnetic stent removal in a nurse-led clinic; A nine-month experience. Ir Med J 2018; 111:687.

21. Sevcenco S, Eredics K, Lusuardi L, et al. Evaluation of pain perception associated with use of the magnetic-end ureteric double $\mathrm{J}$ stent for short-term ureteric stenting. World J Urol. 2018; 36:475-479.

22. Mykylak DJ, Herskowitz M, Glassberg KI. Use of magnetic internal ureteral stents in pediatric urology: retrieval without routine requirement for cystoscopy and general anesthesia. J Urol 1994; 152:976-7.

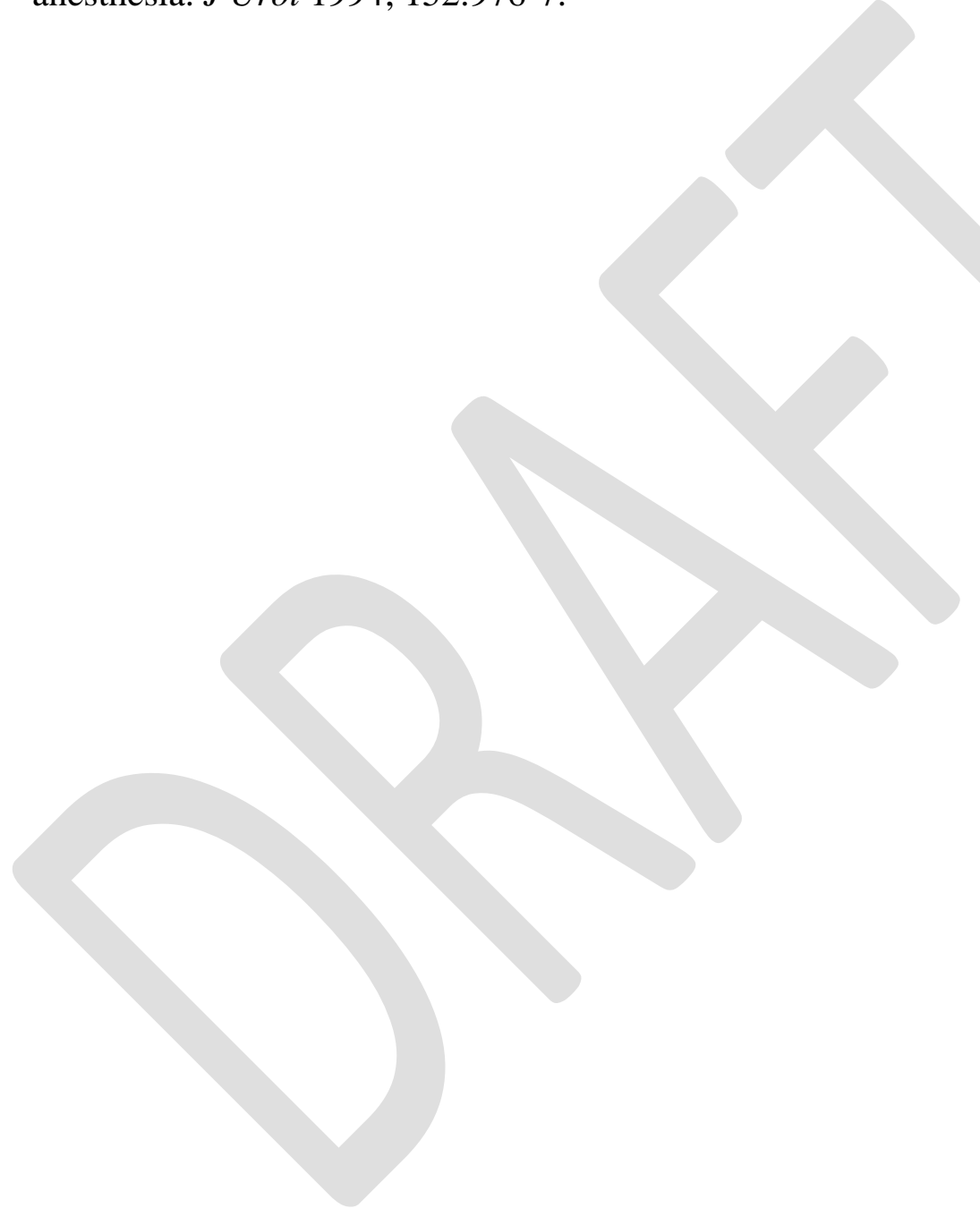


Figures and Tables

Fig. 1. Black-Star ${ }^{\circledR}$ stent design (Urotech, Achenmühle, Germany).

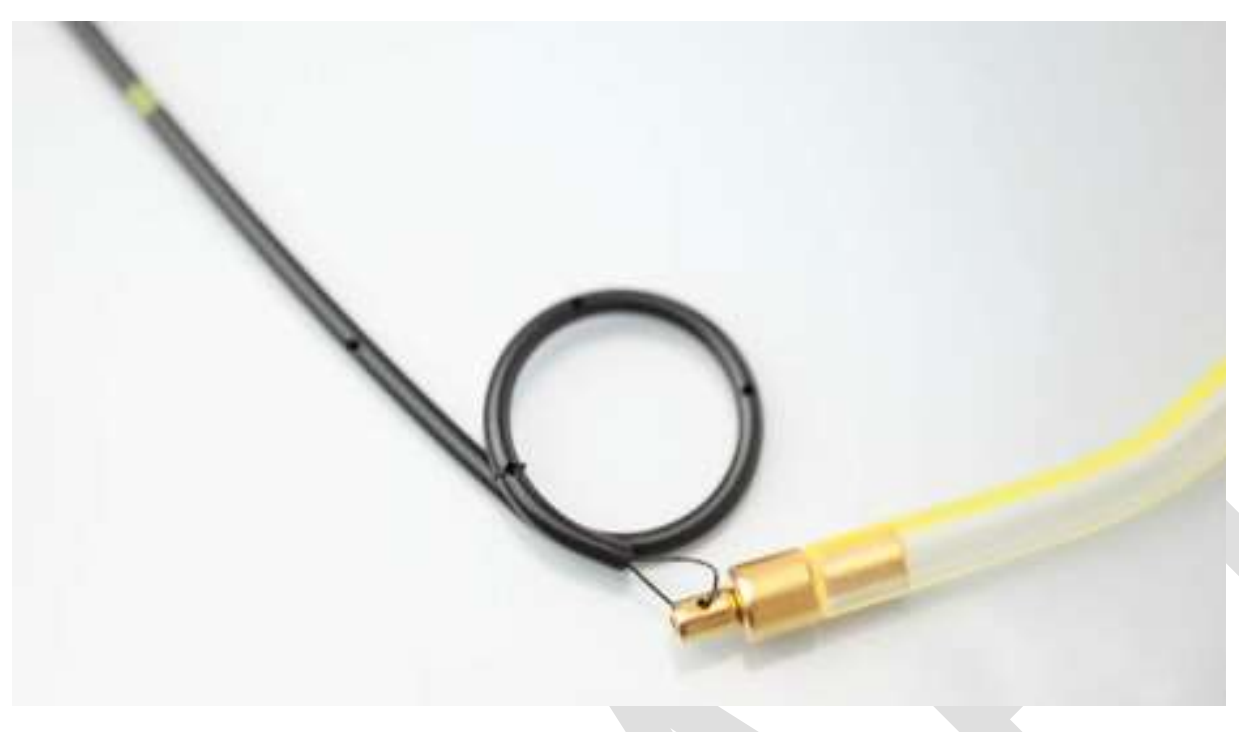

Fig. 2. Black-Star ${ }^{\circledR}$ magnetic separation design (Red Leaf Medical, Inc., Mississauga, ON, Canada).

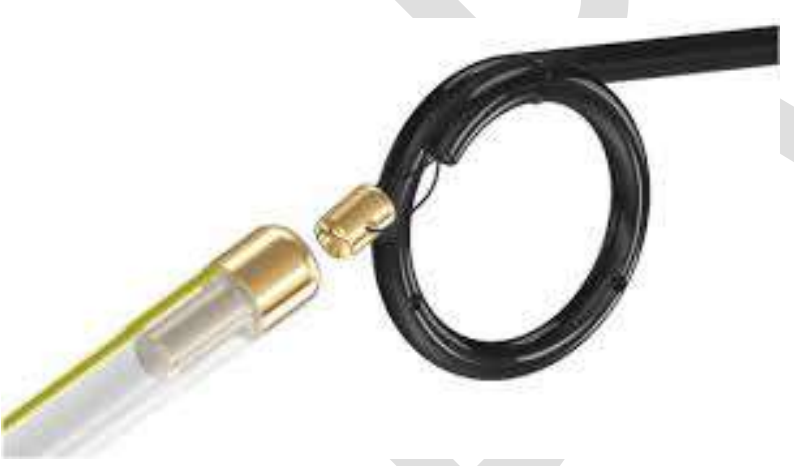




\begin{tabular}{|l|c|c|c|}
\hline \multicolumn{3}{|l|}{ Table 1. Patient demographics and medical comorbidities } \\
\hline Variable & $\begin{array}{c}\text { Black-Star } \\
\text { stent (n=22) }\end{array}$ & $\begin{array}{c}\text { Conventional } \\
\text { stent (n=19) }\end{array}$ & p \\
\hline Age, mean (SD) & $\begin{array}{c}\text { (n.41 } \\
( \pm 11.36)\end{array}$ & $\begin{array}{c}57.16 \\
( \pm 14.96)\end{array}$ & 0.434 \\
\hline Gender, n (\%) & $15(68.2)$ & $12(63.2)$ & 0.735 \\
Male & $7(31.8)$ & $7(36.8)$ & \\
Female & & & \\
\hline Comorbidities, n (\%) & $12(54.5)$ & $9(47.4)$ & 0.647 \\
Hypertension & $5(22.7)$ & $2(10.5)$ & 0.301 \\
Coronary artery disease & $7(31.8)$ & $4(21.1)$ & 0.438 \\
Diabetes mellitus & $0(0)$ & $1(5.3)$ & 0.276 \\
Asthma & $6(27.3)$ & $3(15.8)$ & 0.376 \\
Other & $0(0)$ & $0(0)$ & \\
Previous LUTS, n (\%) & $0(0)$ & $0(0)$ & \\
Urgency & $0(0)$ & $0(0)$ & 0.347 \\
Frequency & $1(4.5)$ & $0(0)$ & \\
Dysuria & $0(0)$ & $0(0)$ & \\
Poor stream & $0(0)$ & $0(0)$ & \\
Straining & $0(0)$ & $0(0)$ & \\
\hline Sense of incomplete voiding & $0(0)$ & $0(0)$ & \\
Hematuria & $0(0)$ & $0(0)$ & \\
Nocturia & $0(0)$ & $0(0)$ & \\
Incontinence & & \\
Pelvic pain & &
\end{tabular}

LUTS: lower urinary tract symptoms; SD: standard deviation. 


\begin{tabular}{|l|c|c|c|}
\hline \multicolumn{4}{|c|}{ Table 2.Mean USSQ scores with stent in situ and 1week post-stent removal } \\
\hline & $\begin{array}{c}\text { Black-Star } \\
\text { mean (SD) }\end{array}$ & $\begin{array}{c}\text { Conventional } \\
\text { mean (SD) }\end{array}$ & p \\
\hline $\begin{array}{l}\text { Urinary index score, stent in } \\
\text { situ }\end{array}$ & $21.48(5.47)$ & $22.47(3.89)$ & 0.515 \\
\hline $\begin{array}{l}\text { Urinary index score, 1-week } \\
\text { post-stent removal }\end{array}$ & $21.75(5.33)$ & $20.64(4.06)$ & 0.481 \\
\hline Global score, stent in situ & $3.58(1.64)$ & $3.21(1.23)$ & 0.439 \\
\hline $\begin{array}{l}\text { Global score, 1-week post- } \\
\text { stent removal }\end{array}$ & $3.60(1.72)$ & $4.20(1.01)$ & 0.255 \\
\hline $\begin{array}{l}\text { General health score, stent in } \\
\text { situ }\end{array}$ & $11.29(3.79)$ & $13.50(4.53)$ & 0.138 \\
\hline $\begin{array}{l}\text { General health score, 1-week } \\
\text { post-stent removal }\end{array}$ & $11.71(4.71)$ & $13.06(4.49)$ & 0.398 \\
\hline Pain index score, stent in situ & $1.75(3.74)$ & $1.68(4.42)$ & 0.960 \\
\hline $\begin{array}{l}\text { Pain index score, 1-week post- } \\
\text { stent removal }\end{array}$ & $3.00(5.47)$ & $2.33(6.83)$ & 0.331 \\
\hline
\end{tabular}

SD: standard deviation.

\begin{tabular}{|l|c|c|}
\hline Table 3. Cost-analysis of Black-Star ${ }^{\circledR}$ and conventional stents per case \\
\hline & $\begin{array}{c}\text { Black-Star }{ }^{\circledR} \text { costs } \\
\text { (\$CAD) }\end{array}$ & $\begin{array}{c}\text { Conventional stent } \\
\text { (osts (\$CAD) }\end{array}$ \\
\hline Cost of stent & 183.96 & 64.85 \\
\hline $\begin{array}{l}\text { Flexible cystoscopy (total cost } \\
\text { including materials and fees) }\end{array}$ & - & 429.28 \\
\hline $\begin{array}{l}\text { Outpatient in-clinic stent removal } \\
\text { (supplies) }\end{array}$ & 6.15 & - \\
\hline Total cost & 190.11 & 495.13 \\
\hline Difference & \multicolumn{2}{|c|}{304.02} \\
\hline
\end{tabular}

